\title{
Sonographic Assessment of Common Bile Duct Diameter among Adults in North Central Nigeria
}

${ }^{1}$ Mohammad H, ${ }^{2}$ Achinge G I, ${ }^{3}$ Eke B A, ${ }^{2}$ Mbaave T P, ${ }^{4}$ Okwori G, ${ }^{5}$ Ojobi J E, ${ }^{6}$ Shaahu V N, ${ }^{7}$ Bitto T T

${ }^{I}$ Department of Radiology Benue State University Teaching Hospital Makurdi, Benue State

${ }^{2}$ Department of medicine Benue State University Teaching Hospital Makurdi, Benue State

${ }^{3}$ Department of surgery Benue State University Teaching Hospital Makurdi, Benue State

${ }^{4}$ Department Radiology Federal Medical Centre Makurdi, Benue State

${ }^{5}$ Department of medicine Federal Medical Centre Makurdi, Benue State

${ }^{6}$ Department of Community Medicine Federal Medical Centre Makurdi, Benue State

${ }^{7}$ Riverside Specialist Clinic, Federal Medical Centre Makurdi, Benue StateCorresponding Author: Mohammad $H$

\begin{abstract}
Background: This was a study to determine the diameter of the common bile duct (CBD) in Nigerian adults. It was intended to be used as a Normogram.

Methods: A hospital based prospective descriptive study was carried out between September and December 2011 on consecutive subjects presenting for ultrasonography at Federal Medical Centre, Makurdi. Ethical approval was obtained from the institute's Ethics Committee. Informed written consent was obtained from each participant. Patients with history of pancreatic, liver or biliary disease or surgery and excessive bowel gas were excluded. Sonographic examination was done using Sonoscape SS1-1000 machine incorporated with electronic calipers. Each patient was scanned in a supine position after an overnight fast. Using Behan and Kazan's description, the diameter of the CBD was measured proximally at the porta hepatis and distally at a site at least $2 \mathrm{~cm}$ superior to the head of the pancreas. The mean of three measurements was recorded. All measurements were done by the same sonologist. Bio data and body mass index of the subjects were recorded. The data was entered into an Excel sheet and analyzed using SPSS Statistics software version 17.0 for mean, median and range.

Results: Two hundred subjects were recruited for this study. One hundred and ninety six (98\%) made up of 61 males $(31.1 \%)$ and 135 females (68.9\%) completed the study. The M: F ratio was 1:2. The median age of the study population was 32.5 years with a range of 15-74 years. CBD diameter in all subjects was between 2-9mm. Majority had CBD diameter of 5-7 $\mathrm{mm}$.

Conclusion: This study showed that normal CBD diameter in this environment was 2 to $9 \mathrm{~mm}$, with a median of 5 to $7 \mathrm{~mm}$.

Key words: Common Bile Duct, Ultrasound, Normogram, correlates.
\end{abstract}

\section{Introduction}

Ultrasonographic assessment of the common bile duct has been used in the evaluation of hepatobiliary disease for over thirty years. ${ }^{1}$

Although, imaging of the CBD may be undertaken with one or a combination of several modalities including computerized tomography (CT), magnetic resonance pancreatography (MRCP), endoscopic retrograde cholangiopancreatography (ERCP), ultrasonography has remained the imaging modality of first choice. Ultrasonography is readily available, non-invasive, relatively cheap and uses non-ionizing radiation. Indeed, extrahepatic biliary obstruction can be demonstrated with a degree of accuracy approaching 100\% with ultrasonography. $^{2}$

Various studies in Europe, Asia, America and Africa have documented CBD diameter among normal populations. ${ }^{3,4,5,6}$ However, a Normogram of CBD diameter among Nigerians is not readily available. The purpose of this study therefore, is to determine the normal CBD diameter in healthy subjects or subjects presenting to hospital with medical conditions other than those of the hepatobiliary system in a defined Nigerian population. This study will contribute to the knowledge on Common bile duct parameters in the Nigerian population.

\section{Methodology}

A hospital based prospective descriptive study was done between September and December 2011 on one hundred and ninety seven (197) subjects. This population consisted of volunteers and patients who were referred from various clinics of the Federal Medical Centre, Makurdi for ultrasonography to evaluate clinical 
conditions other than those with history of Hepatobiliary disease. Excluded from the study were patients with history of pancreatic, liver or biliary disease or surgery. At ultrasound, patients with hepatomegaly (Liver span> $15.5 \mathrm{~cm}$ ) and patients with excessive bowel gas were also excluded.

Sonographic examination was done using Sonoscape SS1-1000 machine incorporated with electronic calipers for accurate measurements. The machine is fitted with 3.5 and $5.2 \mathrm{MHz}$ curvilinear transducers. Each patient was scanned in a supine position on a couch after an overnight fast. After the application of the coupling gel to the upper abdomen, optimum scanning techniques as described by Behan and Kazan were utilized as needed. ${ }^{7}$ These included scanning with the right side of the study subject elevated so that the ultrasound beam is directed posteriomedially. The porta hepatis was scanned longitudinally in deep inspiration through the anterior abdominal wall. Varying the degree of elevation of the right side, the CBD was demonstrated in the porta hepatis. In some subjects, the scan was performed obliquely so that the transducer moved from a more lateral position superiorly to a more medial position inferiorly. The diameter of the CBD was measured at a site at least $2 \mathrm{~cm}$ superior to the head of the pancreas. Measurement was made from inner to inner walls using electronic calipers. The mean of three measurements was recorded in order to reduce intra-observer measurement errors. All measurements were done by the same sonologist to eliminate observer error. The sex, age, height and the weight of the subjects were recorded. The data was entered into an Excel data sheet. The data was analyzed using SPSS Statistics software, version 17.0. Chi Square was used as test of statistics with a P value of less than 0.05 considered significant.

\section{Ethical Considerations}

Ethical approval was obtained from the Federal Medical Center, Makurdi Ethics Committee. Informed written consent was obtained from the subjects after the nature, aims and objectives of the study were explained to the subjects in the language they best understood. Consenting participants signed a consent form or appended their thumb prints appropriately. The study subjects were given an option to opt out of the study if they so wished without affecting any benefits accruing to them.

\section{Results}

Two hundred subjects were recruited for this study. One hundred and ninety six (98\%) completed the study and their results were analyzed. All subjects had normal CBD diameter ranging from 2-9mm. Of the 196 subjects, $61(31.1 \%)$ were males and 135 (68.9\%) females giving an M: F ratio of 1:2. Generally the females had slightly larger CBDs compared to the males; however for both sexes, the majority had CBD diameter of 5-7 $\mathrm{mm}$.

The median age of the study population was 32.5 years with a range of 15-74 years. Generally, majority of subjects had CBD diameter of 5-7 mm irrespective of age group. However, for the age group 65-74 years, majority $7(63.6 \%)$ had CBD diameter of 2-4 mm.

Most, $112(57.1 \%)$ of the subjects had normal BMI (18-24.9 kg/m2). However majority, $11(68.8 \%)$ of those with BMI $30-39.9 \mathrm{~kg} / \mathrm{m} 2$ had CBD diameter of $5-7 \mathrm{~mm}$.

Table 1: CBD stratified by gender

\begin{tabular}{|l|r|r|}
\hline CBD (mm) & Male n (\%) & Female n (\%) \\
\hline $2-4$ & $25(41.0)$ & $57(42.2)$ \\
\hline $5-7$ & $35(57.4)$ & $74(54.8)$ \\
\hline $8-10$ & $1(1.6)$ & $4(3.0)$ \\
\hline Total & $\mathbf{6 1 ( 1 0 0 )}$ & $\mathbf{1 3 5 ( 1 0 0 )}$ \\
\hline
\end{tabular}

Table 2: CBD stratified by age

\begin{tabular}{|l|r|r|r|r|r|r|}
\hline & \multicolumn{7}{|c|}{ Age (years) } \\
\hline & \multicolumn{7}{|c|}{$\mathbf{N}(\%)$} \\
\hline CBD (mm) & $15-24$ & $25-34$ & $35-44$ & $45-54$ & $55-64$ & $65-74$ \\
\hline $2-4$ & $24(43.6)$ & $23(47.9)$ & $8(23.5)$ & $14(46.7)$ & $6(33.3)$ & $7(63.6)$ \\
\hline $5-7$ & $31(56.4)$ & $24(50.0)$ & $23(67.6)$ & $16(53.3)$ & $11(61.1)$ & $4(36.4)$ \\
\hline $8-10$ & $0(0)$ & $1(2.1)$ & $3(8.8)$ & $0(0)$ & $1(5.6)$ & $0(0)$ \\
\hline Total & $\mathbf{5 5 ( 1 0 0 )}$ & $\mathbf{4 8}(\mathbf{1 0 0})$ & $\mathbf{3 4}(\mathbf{1 0 0})$ & $\mathbf{3 0}(\mathbf{1 0 0})$ & $\mathbf{1 8 ( 1 0 0 )}$ & $\mathbf{1 1}(\mathbf{1 0 0})$ \\
\hline
\end{tabular}


Table 3: CBD stratified by BMI

\begin{tabular}{|c|c|c|c|c|c|}
\hline & \multicolumn{5}{|c|}{ BMI $\left(\mathrm{kg} / \mathrm{m}^{2}\right)$} \\
\hline CBD (mm) & $<18$ & $18-24.9$ & $25-29.9$ & $30-39.9$ & $\geq 40$ \\
\hline $2-4$ & $4(66.7)$ & $47(42.0)$ & $24(40.7)$ & $5(31.2)$ & $2(66.7)$ \\
\hline $5-7$ & $2(33.3)$ & $61(54.5)$ & $35(59.3)$ & $11(68.8)$ & $0(0)$ \\
\hline $8-10$ & $0(0)$ & $4(3.6)$ & $0(0)$ & $0(0)$ & $1(33.3)$ \\
\hline Total & $6(100)$ & $112(100)$ & $59(100)$ & $16(100)$ & $3(100)$ \\
\hline
\end{tabular}

\section{Discussion}

The sonographic assessment of CBD via ultrasonography is a cheap, non-invasive and relatively easily available means of evaluating the hepatobiliary system. It is mobile and can be deployed to the point of care as needed. In many parts of Nigeria and other resource limited countries, ultrasonography may be the only method available.

Our result shows a female preponderance. This may not be unrelated to the health seeking behaviour of women, and the fact that majority of individuals referred for ultrasonography in our centre are females presenting mostly for gynaecological scans.

Our study population consists mainly of young adult subjects with a median age of 32.5 years. Only 11 study subjects were 65 years or older. Generally, in African setting the elderly people are not economically empowered hence are unable to access health care. Some even think it may be an unnecessary burden on their children or care giver. Also, most elderly people who presented for ultrasonography had some background disorders that excluded them from the study.

The range of CBD diameter in this apparently normal population seeking health in our centre was 2$9 \mathrm{~mm}$. Wu and co-workers have reported an upper limit of normal CBD diameter to be $10 \mathrm{~mm}^{8}$. However, other workers have reported the normal upper limit to be between 6 to $7 \mathrm{~mm}^{9,10,11}$. More than $50 \%$ of both male and female participants in this study had a CBD diameter of $5-7 \mathrm{~mm}$. This finding is similar with that documented by other researchers who have reported values of $4-8 \mathrm{~mm}^{12,13}$.

This study did not show any consistent pattern between age and the CBD diameter. Kaude reported a similar finding in his study ${ }^{14}$. Other studies have however, observed an increase in the diameter of the CBD with age ${ }^{1,6}$. The finding in our study may be explained by the relatively small proportion of respondents that were 65 years or older.

Generally, increasing BMI did not appear to influence CBD diameter in this study. This contradicts the observation by Adibi and Givechian who noted a positive correlation between increasing BMI and CBD ${ }^{1}$.

\section{Conclusion}

This study showed that normal CBD diameter in this environment is 2 to $9 \mathrm{~mm}$, with a median of 5 to 7 $\mathrm{mm}$. This is the first of such a study in our environment. A multicenter study with a larger study population is required to better define the $\mathrm{CBD}$ diameter and its associations.

\section{References:}

[1]. Adibi A, Givechian B. Diameter of common bile duct: what are the predicting factors? JRMS 2007; 12 (3): $121-124$

[2]. Lokich JJ, Kane RA, Harrison DA, McDermott WV. Biliary tract obstruction secondary to cancer: management guidelines and selected literature. JCO 1987; 5 (6): 969-981.

[3]. Kaim A, Steinke K, Frank M, Enriquez R, Bongartz G et al. Diameter of the common bile duct in the elderly patient: measurement by ultrasound. Eur Radiol 1998; 8(8):1413-1415.

[4]. Park NC, Lee JS, Cho SW, Shim CS. The measurement of size of extrahepatic bile ducts in normal control, patients with cholelithiasis and postcholecystectomy patients. Korean J gastroenterol 1987; 19:572-576.

[5]. Bowie JD. What is the upper limit of normal for the common bile duct on ultrasound: how much do you want it to be? Am $\mathbf{J}$ Gstroenterol 2000; 95(4):897-900.

[6]. Admassie D. Ultrasound assessment of common bile duct diameter in Tikur Anbessa Hospital, Addis Ababa, Ethiopia. Ethiop Med J. 2008 Oct; 46(4):391-5.

[7]. Behan M, Kazam M. Sonography of the Common Bile Duct: Value of Right Anterior Oblique View. Am J Roentgenol 1978; 130:701709.

[8]. Wu CC, Chen CY, Ho YH: Effect of aging on the common bile duct: Areal time ultrasound study. J Clin Ultrasound 12:473, 1984.

[9]. Parulekar SG: Ultrasound evaluation of the common bile duct size. Radiology 133:703, 1979.

[10]. Ferruci JT, Adson MA, Mueller PR, et al: Advances in the radiology of jaundice; a symposium and review. AJR 141:1, 1983.

[11]. Nierderau C, Mueller J, Sonnerberg A, et al: Extrahepatic bile ducts in healthy subjects, in patients with cholelithiasis, and in postcholecystectomy patients: A prospective ultrasonic study. J Clin Ultrasound 11:23, 1983.

[12]. Dewburry KC. Visualization of normal biliary ducts with ultrasound. Br J Radiol 1980; 53:774.

[13]. Cooperberg PL, Li D, Wong P, et al. Accuracy of common hepatic duct size in the evaluation of extrahepatic biliary obstruction. Radiology 1980; 135:141-144.

[14]. Kaude KV. The width of the common bile duct in relation to age and stone. Eur J Radiol 3:115, 1983 